\title{
SOM Training Approach for OFDM Receiver
}

\author{
Shubhangi Rathkanthiwar, Chandrashekhar Dethe, and Kishore Kulat
}

\begin{abstract}
This paper presents a novel approach for performance improvement in WLAN receiver employing Orthogonal frequency division multiplexing (OFDM) technology. As an effective technology for wireless communications, OFDM is an important multicarrier modulation method that offers high spectral efficiency, multipath delay spread tolerance, and immunity to frequency selective fading. However, some challenging issues still remain unresolved in OFDM based system design, such as its high peak to average power ratio (PAPR). Methods used to reduce PAPR factor carry several disadvantages. Neural approach presented in this paper is the new method which provides function approximation method. WLAN receiver under consideration is Hiperlan/2. Modulation method is $16 Q A M$ with code rate $3 / 4$ and AWGN channel model is used. Self organizing map (SOM) and parameterless self organizing map (PLSOM) structures are used to improve bit error rate (BER) performance, which was degraded because of nonlinear distortions due to high PAPR factor.
\end{abstract}

Index Terms-High power amplifiers, neural networks, self organizing map, orthogonal frequency division multiplexing.

\section{INTRODUCTION (OFDM SYSTEMS PAPR FACTOR)}

Most of the radio systems employ the HPA in the transmitter to obtain sufficient transmission power. To provide maximum output power efficiency, the HPA is usually operated at or near the saturation region. Nonlinear characteristics of the HPA are very sensitive to the variation in signal amplitudes. Variations of OFDM signal amplitudes are very wide with high PAPR. Therefore, HPA will introduce inter-modulation between the different subcarriers and introduce additional interference into the systems due to high PAPR of OFDM signals. This additional interference leads to an increase in BER [1]-[4].

An OFDM symbol is a sum of $\mathrm{N}$ independent symbols mapped on $\mathrm{N}$ different sub-channels with $1 / \mathrm{T}$ frequency separation. $\mathrm{T}$ is the OFDM symbol period. Discrete time domain samples $b^{\mathrm{i}}=\left(b_{0}{ }^{\mathrm{i},}, b_{1}{ }^{\mathrm{i}} \ldots \ldots b_{\mathrm{N}-1}{ }^{\mathrm{i}}\right)$ to be transmitted are obtained via $\mathrm{N}$ point IFFT from complex 16 QAM symbols in this case. A block of QAM symbols is given by [5]-[8], $a^{\mathrm{i}}=\left(a_{0}{ }^{\mathrm{i}}, a_{2}{ }^{\mathrm{i}}, \ldots \ldots a_{\mathrm{N}-1}^{\mathrm{i}}\right)$.

Mathematically an OFDM symbol can be represented by equation (1)

$$
b_{n}^{i}=\frac{1}{\sqrt{N}} \sum_{m=0}^{N-1} a_{m}^{i} e^{J 2 \pi m n / N}
$$

Manuscript received October 15, 2012; revised November 20, 2012.

S. Rathkanthiwar is with Department of Electronics Engineering Yeshwantrao Chavan College of Engineering, Nagpur, India (e-mail: svr_1967@yahoo.com).

C. Dethe is with Priyadarshini Institute of Enginnering and Technology, Nagpur, India. (e-mail: cgdethe@yahoo.com).

K. Kulat is with Electronics Engineering Department Vishvesaraya National Institute of Technology (e-mail: kishore_kulat@yahoo.com) where $a_{m}^{i}$ is the QAM data symbol sent on $\mathrm{m}^{\text {th }}$ subcarrier of $\mathrm{i}^{\text {th }}$ OFDM symbol. For large number of subcarriers, time domain samples $b_{m}^{i}$ have a zero mean Guassian distribution, as they are weighted sum of independent, identically distributed random variables. Some of these time domain samples, which lie at the tail of the distribution curve, gain high magnitudes and cause high PAPR of the system. Mathematically, PAPR of OFDM block of digital samples $\mathrm{b}=\left(\mathrm{b}_{0}, \mathrm{~b}_{1}, \ldots \mathrm{b}_{\mathrm{N}-1}\right)$ is given by equation $(2)$.

$$
\operatorname{PAPR}(b)=\frac{\max _{0<n<N-1}\left|b_{n}^{2}\right|}{E\left\{|| b_{n}^{2} \|\right\} / N}
$$

\section{SOM ALGORITHM}

SOM variant is the Guassian neighborhood, Euclidean distance and neighborhood size [9].

An input $x(t)$ is presented at time $t$. Winning node $c(t)$, i.e. the weight vector that most closely matches the input at time $t$, is selected using (3) [10].

$$
c(t)=\arg \min _{t}\left(\|\| x(t)-w_{i}(t)_{2} \|\right)
$$

where $w_{i}(t)$ is the weight vector of node $i$ at time $t .\|\|_{2}$ denotes $L^{2}$ norm or $\mathrm{n}$ dimensional Euclidean distance. Weights of all nodes are then updated using equation (4)

$$
\begin{gathered}
w_{i}(t+1)=w_{i}(t)+\Delta w_{i}(t) \\
\Delta w_{i}(t)=\alpha(t) h_{c, i}(t)\left\lceil x(t)-w_{i}\right\rceil(t) \\
h_{c, i}(t)=e \frac{-d(i, c)^{2}}{\beta(t)^{2}}
\end{gathered}
$$

where $h_{c, i}(t)$ is the neighborhood function and is a scaling function centered on the winning node $c$ decreasing in all directions from it. $d(i, c)$ is the Euclidean distance from node $i$ to the winning node $c$ in the node grid. $\alpha(t)$ is the learning rate at time $t$ and $\beta(t)$ is the neighborhood size at time $t$

Learning rate $\alpha$ and neighborhood size $\beta$ are decreased in accordance with the annealing scheme.

One possible annealing scheme for the decrease of learning rate and neighborhood size is given by (7) and (8).

$$
\alpha(t+1)=\alpha(t) \delta_{\alpha}
$$

And

$$
\beta(t+1)=\beta(t) \delta_{\beta}
$$


where $0<\delta_{\alpha}<1$ and $0<\delta_{\beta}<1$.

Here $\delta_{\alpha}$ and $\delta_{\beta}$ are the scaling constants.

These steps are repeated until some preset condition is met, after some iterations or when measurement error reaches certain level. Density of the nodes is proportional to input samples.

Unfortunately, this unsupervised learning is dependent on two annealing schemes, one for the learning rate and one for the neighborhood size. There is no firm theoretical basis for determining the correct type and parameters for these annealing schemes, so they must be determined empirically. Furthermore, since these annealing schemes are time dependent, they prevent the SOM from assimilating new information, once the training is complete. It is not in tune with adaptive capabilities of SOM we expect.

Parameterless self organizing map is a neural network, based on SOM that eliminates need for a learning rate and neighborhood size. Fundamental difference between SOM and PLSOM is that, while a SOM depends on learning rate and neighborhood size to decrease over time, as a function of number of iterations of the learning algorithm, PLSOM calculates these values based on the local quadratic fitting error of the map to the input space. This allows the map to make large adjustments in response to the unfamiliar inputs i.e. the inputs, that are not well mapped while not making large changes in response to the inputs it is well adjusted to. Fitting error is based on the normalized distance from input to the weight vector of the winning node in the input space. This value is computed in any case, hence this mechanism can be implemented without inducing noteworthy increases in the computational load of the map or hindering parallelized implementation.

\section{PLSOM ALGORITHM}

Fundamental idea of PLSOM is that the amplitude and extent of weight updates are not dependent on iteration number, but how good the fit is, we calculate a scaling variable $\varepsilon$ which is then used to scale the weight update, which can be defined through following equations (9) and (10).

$$
\varepsilon(t)=\frac{\left\|x(t)-w_{c}(t)\right\|_{2}}{r(t)}
$$

where

$$
\begin{gathered}
r(t)=\max \left(\left\|x(t)-w_{c}(t)\right\|, r(t-1)\right. \\
r(0)=\left\|x(0)-w_{c}(t)\right\|_{2} .
\end{gathered}
$$

$\varepsilon(t)$ is best understood as the normalized Euclidean distance from the input vector at time $t$ to the closest weight vector. If this variable is large, network fits the input data poorly and needs a large adjustment. Conversely, if $\varepsilon$ is small, the fit is likely to already be satisfactory for that input and no large update is necessary.

Algorithm for PLSOM uses a neighborhood size determined by $\varepsilon$ thus replacing the equation governing the annealing of neighborhood with $\beta(t)=$ cons $\tan t \forall t$. $\beta$ is scaled by $\varepsilon(t)$ in the manner of equation (11), giving $\Theta(\varepsilon(t))$ the scaling variable for neighborhood function (12)

$$
\Theta(\varepsilon(t))=\beta(t)
$$

where

$$
\Theta(\varepsilon(t)) \geq \theta_{\min }
$$

\section{Simulations}

Simulations are carried out using MATLAB SIMULINK model 16 QAM modulation schemes for $3 / 4$ code rate for AWGN channel. Hiperlan/2 Simulink model with TWTA (Saleh model) and SSPA (Rapp's model) has been simulated for four cases.

1) Hiperlan/2 without neural block with AWGN channel

2) Hiperlan/2 without neural block with Rayleigh fading channel

3) Hiperlan/2 with SOM block

4) Hiperlan/2 with PLSOM block

Self organizing map recognize groups of similar input vectors in such a way that neurons physically close to each other respond to similar input vectors. It classifies input vectors according to how they are grouped in the input space. Neurons are arranged originally in physical positions according to topological functions. The function newsom creates a self organizing map with the following parameters.

1) PR: The matrix of min and max values

2) Di: The size of ith layer dimensions

3) TFCN: The topology function

4) DFCN: The distance function

5) OLR: Ordering phase learning rate

6) OSTEPS: Ordering phase steps

7) TLR: Tuning phase learning rate

8) TND: Tuning phase neighborhood distance

This returns a new SOM. For this paper, SOM is trained with 1000 epochs and the input vectors are plotted with the map that formed the SOM weights. SOM consists of a single layer with 'negdist' weight function, 'netsum' net input function and 'compet' transfer function. The layer has a weight from the input but no bias. The weight was initialized with 'midpoint'. Adaptation was done with the function 'trainr' and the weights were updated with the algorithm 'learnsom'. The distance function used is 'dist' which calculates the Euclidean distance from home neuron to any other neuron. All the neighborhoods for a $S$ neuron layer map are represented by S X S matrix of distances. 'Gridtop' topology starts with the neuron in the rectangular grid. Weights of winning neurons are adjusted using Kohonen's rule. The rule allows the weights of neurons to learn an input vector. Thus the neuron, whose weight vector was closest to the input vector was updated to be even closer.

PLSOM completely eliminates selection of learning rate, the annealing rate and the annealing scheme of learning rate and the neighborhood size. It also decreases the number of iterations required to get a stable and ordered map. It has been proved that in 600 epochs we are getting the desired result. 
TABLE I: SIMULATION PARAMETERS FOR HIPERLAN/2 WITH SALEH MODEL

\begin{tabular}{|l|l|}
\hline Parameter & Value/ Description \\
\hline $\begin{array}{l}\text { WLAN Model for } \\
\text { simulation }\end{array}$ & Hiperlan/2 \\
\hline Modulation scheme & 16 QAM \\
\hline Code rate & $3 / 4$ \\
\hline Puncture vector & 52 \\
\hline Number of sub carriers & 64 \\
\hline FFT size & 16 \\
\hline GI length parameter & $\begin{array}{l}\text { AWGN/ Rayleigh } \\
\text { fading }\end{array}$ \\
\hline Channel model & 2.1587 \\
\hline $\begin{array}{l}\text { AM/AM } \\
\text { alpha }\end{array}$ & 1.1517 \\
\hline AM/AM parameter beta & 4.0033 \\
\hline AM/PM parameter alpha & 9.1040 \\
\hline AM/PM parameter beta &
\end{tabular}

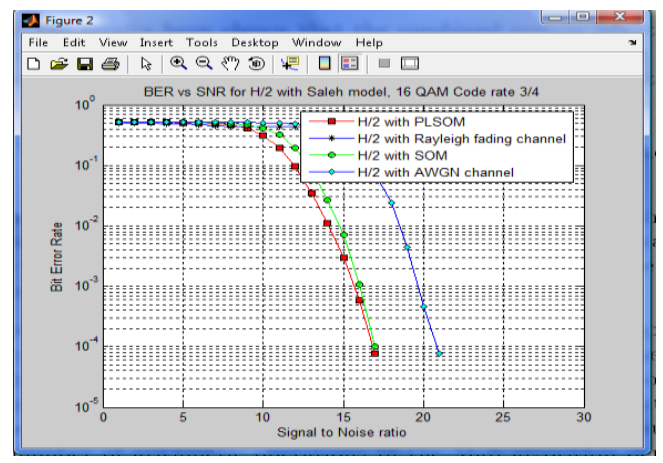

Fig. 6. BER plot for Hiperlan/2 with saleh model

Simulation setting for getting BER plot for Hiperlan/2 with Rapp's model is given in table 2 and the BER plot is shown in figure 7 .

TABLE II: SIMULATION PARAMETERS FOR HIPERLAN/2 WITH RAPP'S MODEL

\begin{tabular}{|l|l|}
\hline Parameter & Value/ Description \\
\hline $\begin{array}{l}\text { WLAN Model for } \\
\text { simulation }\end{array}$ & Hiperlan/2 \\
\hline Modulation scheme & 16 QAM \\
\hline Code rate & $3 / 4$ \\
\hline Puncture vector & \\
\hline Number of sub carriers & 52 \\
\hline FFT size & 64 \\
\hline GI length & 16 \\
\hline Channel model & $\begin{array}{l}\text { AWGN/ Rayleigh } \\
\text { fading }\end{array}$ \\
\hline Smoothness factor & 2 \\
\hline
\end{tabular}

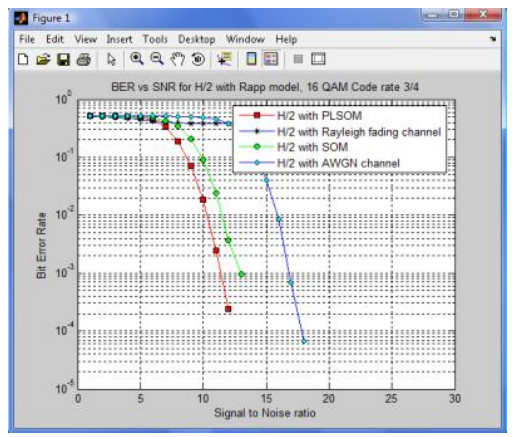

Fig. 7. BER plot for Hiperlan/2 with Rapp's model

For our case, we have trained MATLAB SOM variants, SOM and PLSOM with identical input data. We have taken pseudorandom two dimensional input data eliminating the need to store training data. Since the training data is uniformly distributed in the input space, perfect distribution of weight vectors would be an evenly spaced grid with a narrow margin along the edges of the input space. Each weight vector would map an evenly sized area of the input space.

Simulation setting for getting BER plot for Hiperlan/2 with Saleh model is given in table 1 and the BER plot is shown in Fig. 6.

\section{CONCLUSION}

This paper presents results of simulations run on neural network based Hiperlan/2 WLAN receiver structure in fading multipath environment. Hiperlan/2 uses OFDM as multiplexing scheme, which has a main drawback of having high peak to average power ratio. Most of the signal components are amplified in saturation region of the high power amplifiers used at the transmitter end causing nonlinear distortions. For this paper, simulations are run for Hiperlan/2 with HPA models like Saleh model (TWTA) and Rapp's model (SSPA). Modulation method is 16 QAM, and code rate is $3 / 4$. Channel models are AWGN and Rayleigh fading. SOM and PLSOM blocks act as adaptive decision devices that not only compensate for nonlinear distortions but also follow up error signals. BER vs SNR plots clearly indicate the better performance of new neural based Hiperlan/2 WLAN receiver structure.

\section{REFERENCES}

[1] H. Zareian and V. Vakili, "Analysis of nonlinear distortion using orthogonal polynomials HPA model," IJCSNS- International journal of computer science and network security, vol. 8, no. 1, Jan 2008, pp. $164-165$

[2] V. G. Jeon and K. H. Chang, "An adaptive data predistorter for compensation on nonlinear distortions in OFDM systems," IEEE Transactions on Communications, vol. 45, no. 10, Oct. 1997, pp. 1167-1171.

[3] H. W. Kang, Y. S. Cho, and D. H. Youn, "On Compensating nonlinear distortions of an OFDM system using an efficient adaptive predistorter," IEEE Transactions on Communications, vol. 47, no. 4, April 1999, pp. 522-526

[4] H. D. Han and P. A. Voecher, Simultaneous Predistortion and nonlinear detection of nonlinearly distorted OFDM signals.

[5] A. Eltholth and A. Mekhail, Modelling effects of clipping and power amplifier nonlinearities on OFDM systems.

[6] A. Eltholth and A. Mekhail, "Peak to average power ratio reduction in OFDM systems using Huffman coding," WASET, in Proc. of world academy of science, Engg and technology, vol. 33, Sep. 2008, ISSN 2070-3740, pp. 266-270

[7] S. Lekvaranyu and Y. Miyanaga, "ML detection with symbol estimation for nonlinear distortions of OFDM signal," WASET , in proceeding of world academy of science, Engg and technology, vol.233, Jan. 2005, ISSN 1304-6884, pp. 46-49

[8] B. Sklaar, Fading channels.

[9] S. Haykin, Neural Networks, a comprehensive foundation, pHI, second edition.

[10] E. Berglund and J. Sitte, "Parameterless self organizing map algorithm," in Proceedings of IEEE tranactions on neural networks, vol.17, Issue 2, March 2006, pp. 305-316.

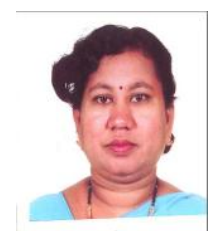

Shubhangi Rathkanthiwar is working as Associate Professor in department of Electronics Engineering, in Yeshwantrao Chavan College of Engineering, Nagpur, India. She has obtained her BE (Electronics and Telecommunications) degree from Marathwada University, India, M. Tech. (Electronics) from Nagpur University, India and submitted her Ph.D. in Rashtrasant Tukdoji Maharaj Nagpur University, India. Her area of expertise are Wireless communications, Neural Networks and Embedded 
systems. She has 46 Conference papers and 5 Journal papers in her credit. She is life menber of Indian Society for Technical Education, and IAENG, i.e. International association of Engineers and associate member of Institution of Engineer, India.

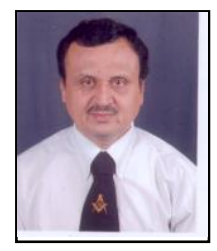

Kishore D. Kulat completed his degrees in Electrical Engineering, BE in 1980, from VRCE (at present VNIT) Nagpur and ME degree in 1984 from VJTI, Mumbai, India. He completed his Ph.D. degree in Electronics Engineering, in the year 2003 from VNIT, Nagpur. Having a total experience of more than 25 years, he is currently associated with VNIT, as Professor and Head of Electronics Department. With his profound knowledge \& experience in his field he is guiding around 15 research scholars for their doctoral degree. Ten have been awarded the $\mathrm{Ph}$. D. degree. He has published around 25 Journal Papers, more than 50 papers in International Conferences \& more than 100 have been published in National Conferences. Has worked as Reviewer for many National \& International Conferences. He is a member of Board of Studies for Electronics
Engineering, RTM Nagpur University for last 10years. He is member of Professional societies like IETE, IEI and ISTE. With all his faith in God, Dr. K. D. Kulat believes in achieving excellence through the process of continuous upgradation.

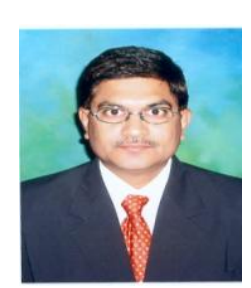

Chandrasekhar G. Dethe received his M.Tech. degree in Electronics Engineering from VNIT, Nagpur, and received his Ph.D. degree from Amravati University., Amravati. He worked as an Assistant Professor at SSGM, College of Engineering Shegaon from 1999 to 2005, later on he joined as a professor at YCCE, Nagpur. $\mathrm{He}$ is currently working as a principal of PIET, Nagpur. His fileld of specialization includes digita communication, Data network, Signal Processing. His papers are published in National and International Journals including IEEE proceedings. He is a fellow of IE and IETE and Life member of ISTE. 